\title{
A Case Report of Swelling of the Left Side of the Face Invading Masseter - An Enigma
}

\author{
Dr. Nagalaxmi $\mathrm{V}^{1}$, Dr. Naga Jyothi $\mathrm{M}^{2}$, Dr. Sridevi. $\mathrm{U}^{3}$, Dr. Kotya Naik M. ${ }^{4}$ \\ 1,2,3,4 (Dept. of Oral Medicine and Radiology, Sri Sai college of Dental Surgery,India)
}

\begin{abstract}
Keratocystic Odontogenic Tumor has been defined by the World Health Organization as a benign intraosseous neoplasm of odontogenic origin with characteristic lining of the parakeratinized squamous epithelium. Due to variable features of this lesion pertaining to clinical and biologic behaviour, this tumor is been studied extensively even today with respect to its molecular aspects to resolve many enigmatic features of it to arrive at a more successful treatment aspects at the genetic level. This paper aims to present a case of nonsyndromic keratocystic odontogenic tumor in a 50 year old male that involved the entire ascending ramus of the mandible not associated with the impacted tooth invading to the masseter muscle by destroying the bony cortices of the left mandible. The presentation of this case resembles a neoplastic variant with aggressive features causing difficulty in complete excision of the lesion due to ill-defined margins with soft tissue extension. Key words: Keratocystic odontogenic tumor, Benign cystic neoplasm, Recurrence.
\end{abstract}

\section{Introduction:}

Odontogenic tumors occur with a relatively high incidence in the jaws with intra-osseous and extraosseous variants compared to other pathologies of head and neck. These tumours are derived from epithelial, ectomesenchymal and/or mesenchymal elements that still are, or have been, part of the tooth forming apparatus and hence therefore, are found exclusively within the maxillofacial skeleton (intraosseous or centrally located), or in the soft tissue (gingiva) overlying tooth-bearing areas or alveolar mucosa in edentulous regions (extraosseous or peripherally located). The tumours may be generated at any stage in the life of an individual. All the odontogenic tumors causing the jaw diseases possess specific biologic behavior based on which they are classified into benign, malignant and non-neoplastic varieties although other non-odontogenic tumors are also contributory to the various established and emerging lesions of the maxillofacial region. Keratocystic odontogenic tumor was earlier called as odontogenic keratocyst which is classified under odontogenic cysts but the clinico-pathological profile of this lesion closely resembles the benign neoplasm with aggressive behavior and malignant potential, henceforth changed its nomenclature from the cyst to tumor as a result of the efforts and research reports by many clinicians and pathologists and revised classifications[1,2].

Keratocystic odontogenic tumor is defined as "A benign uni- or multicystic, intraosseous tumour of odontogenic origin, with a characteristic lining of parakeratinized stratified squamous epithelium and potentially aggressive, infiltrative behaviour. It may be solitary or multiple. The latter is usually one of the stigmata of the inherited nevoid basal cell carcinoma syndrome (NBCCS) which is inherited as an autosomal dominant trait with variable expressivity[2,3]. There has been a great deal of interest in the odontogenic keratocyst (OKC) since it became apparent that it may grow to a large size before it manifests clinically and thus, unlike other jaw cysts, it has a particular tendency to recur following surgical treatment. OKC may be a benign cystic neoplasm where Shear in 2003 called it as keratocystoma followed by "keratinising cystic odontogenic tumour (KCOT)" (Reichart and Philipsen, 2004) and Philipsen in 2005 as keratocystic odontogenic tumor[4].

KCOT has been defined by the World Health Organization as a benign intraosseous neoplasm of odontogenic origin with characteristic lining of the parakeratinized squamous epithelium[4]. OKC is known for its rapid growth and its tendency to invade the adjacent tissues including bone. It has a high recurrence rate of 16 to $30 \%$. OKC can present as unilocular and multilocular tumour. This paper aims to present a case of nonsyndromic keratocystic odontogenic tumor in a 50 year old male patient, that involved the entire ascending ramus of the mandible, not associated with the impacted tooth and invading to the masseter muscle by destroying the bony cortices of the left ramus of the mandible.

\section{Case Report:}

A 50 year old male patient was reported to the department of Oral Medicine and Radiology with a chief complaint of severe pain and swelling involving the lower left posterior jaw region associated with restricted mouth opening since 1 month subsequent to which he was unable to swallow the food normally. He noticed a swelling 3 months back in the affected region of the mandible which was asymptomatic with no intraoral signs of pain, swelling or drainage but he fortunately noticed it increasing gradually in size to the present size as shown in the [Figure 1]. Pain was gradual in onset, throbbing in nature, intermittent, moderate in 
intensity, aggravated on chewing food associated with trismus and denied any history of trauma along with a non-contributory medical history. On extra-oral examination, revealed diffuse swelling in the middle one-third of the face on left side anterior to left tragus of ear involving the entire ramus adjoining massetric region in the area of parotid gland and inferiorly extending to the lower border of the mandible with a size of approx. $5 \times 5$ $\mathrm{cm}$. resulting in gross facial asymmetry. Overlying skin shows no evidence of observable pulsations and draining sinus. The non-assisted mouth opening was approx. $1.5 \mathrm{~cm}$ seen in [Figure 2] and there was severe pain on assisted mouth opening $(1.8 \mathrm{~cm})$. On palpation the swelling was tender to touch, firm in consistency with no local rise in temperature, non-reducible, non-fluctuant, non-compressible, non-pulsatile with no visible discharge. A single left tender submandibular lymph node of size approx. $1 \times 1 \mathrm{~cm}$ in size, firm, mobile was palpable. On intraoral examination there was no evidence of obliteration of the upper and lower buccal vestibules, ulcerations or draining sinuses [Figure 3]. On palpation, there was mild expansion on the lingual aspect of the mandibular ramus and tenderness was elicited on palpating the left masseter and lateral pterygoid muscle, crepitus was not felt. Full complement of teeth were present. Neither displacement nor mobility of the teeth in the respective quadrant was seen. On aspiration with Wide bore needle, a thick blood-tinged creamish white viscous precipitate around $5 \mathrm{ml}$ was aspirated. Increase in the mouth opening was appreciated upon aspiration. [Figure 4]. Based on the above findings of history, clinical examination and aspiration content, provisional diagnosis of Odontogenic keratocyst involving left ramus of the mandible was considered.

\section{Differential Diagnosis:}

Differential diagnosis of Ameloblastoma, Dentigerous cyst due to the similar site specificity, Traumatic bone cyst and Aneurysmal bone cyst due to similar clinical features but there was no history of trauma. Also Odontogenic myxoma, Central giant cell granuloma, Calcifying epithelial odontogenic tumor, Central hemangioma were considered.

\section{Investigations:}

Routine laboratory blood investigations showed no abnormality in the values. Orhtopantomograph shows a well-defined multilocular radiolucency intervening with 2 to 3 faint radio-opaque septae extending posteriorly from distal aspect of 38 region to, involving entire medio-lateral aspect of the ramus including coronoid and condylar processes which is surrounded by well-defined radioopaque sclerotic margins with no evidence of displacement and resorption of the teeth and with intact anterior border of the ascending ramus [Figure 5]. A lateral oblique view of ramus of mandible shows clear multilocularity with cloudy and hazy cystic lumen [Figure 6]. Computed Tomography along with reconstructed images showed a large expansile lytic lesion with cortical perforation, invasion of the lesion into left masseter muscle and parapharyngeal space [Figure $7,8,9 \&$ 10]. Incisional biopsy was done through a $1 \times 1 \mathrm{~cm}$ bony window in the medial aspect of the ramus, $2 \mathrm{~cm}$ behind the third molar and sent to histopathological examination which shows parakeratinized squamous epithelium that is corrugated and 6-10 cell layered in thickness with palisaded, polarized, tall columnar cells in the basal layers containing hyperchromatic nuclei and the absence of rete pegs. The connective tissue consists of plasma cells, lymphocytes and extravasated red blood cells [Figure 11].

Based on the above clinical, radiological and histopathological features, the case was diagnosed as Keratocystic odontogenic tumor.

\section{Treatment:}

Patient was thoroughly educated about clinical severity of the condition followed by the surgical planning after ensuring thorough general examination where the complete resection of the left ramus of the mandible was planned.

\section{Discussion:}

Although Keratocystic odontogenic tumor is well studied, there remains many unanswered questions relating to its clinico-pathologic behavior, high recurrence rates as well as its treatment strategies. In the mid 1950s, it was unanimously declared that the primordial cyst and keratocystic odontogenic tumor can be used synonymously where in the 1992 WHO classification system listed odontogenic keratocyst as the preferred designation over the primordial cyst[5].

Odontogenic keratocysts are fairly common. It has been determined that about $10 \%$ of dentigerous and almost $30 \%$ of primordial cysts keratinize, which is causative factor for its recurrence. It is not known why the linings of some dentigerous and primordial cysts keratinize. Similarly, it is not known why keratinization makes these cysts more aggressive than their non-keratinizing counterparts.

KOT accounts for about $3 \%$ to $11 \%$ of all the odontogenic cysts. The prevalence of OKC varies from $11.6 \%$ to $19.1 \%[6]$. The epidemiological profile of the odontogenic tumors placed the KOT in the first place 
shifting the odontoma to the second place in the frequency of occurrence[7]. This data helps in awakening the oral clinicians in developing new approaches in managing these tumors and avoiding their recurrences.

OKCs occur over a wide age range and cases have been recorded as early as the first decade and as late as the ninth. In most series there has been a pronounced peak frequency in the second and third decades, with figures ranging from $40 \%$ to $60 \%$ of patients being in this age group with slight male predilection, male : female ratio was $1.68: 1^{[4]}$ with the marked tendency to involve mandible in $65-83 \%$ of the cases mostly involving posterior body and ascending ramus as seen in our case.

Keratocystic odontogenic tumor (KOT) is a benign, uni or multicystic, intraosseous tumor that presents aggressive, infiltrative behavior. It may occur as Solid-cystic, peripheral and intraosseous variants of sporadic KOT. Rare cases of parakeratinized cyst arising from the soft tissue, in the skin, temporomandibular joint [8] are also reported. The KCOT behaves like a tumor in many ways, for e.g. involvement of large areas of the bone, high recurrence rate, distinctive histopathological features of the lesion, dysregulation of the PTCH (patched) gene in both Nevoid basal cell carcinoma syndrome associated and sporadic odontogenic keratocysts, etc. Truly, cases of carcinoma arising in KCOT have been reported[9]. Our case is non-syndromic variety with an aggressive and neoplastic behaviour as observed in CT images.

Most often early lesions remain asymptomatic and do not reveal gross clinical signs and symptoms which usually manifest after the lesion grows to a considerably large size involving the maxillary sinus and the entire ascending ramus, including the condylar and coronoid processes as in our case. This occurs because the OKC tends to extend in the medullary cavity and clinically observable expansion of the bone occurs late. Patients with OKCs complain of pain, swelling or discharge. Occasionally, they experience paraesthesia of the lower lip or teeth. Some are unaware of the lesions until they develop pathological fractures. In our case there are no symptoms of paraesthesia but there is pain in the left ramus region associated with trismus and no evidence of gross intraoral swelling. When associated with teeth, the enlarging tumor causes displacement of the teeth, extrusion and divergence of erupted teeth and rarely resorption. Large and aggressive lesions involving maxillary sinus leads to destruction of the sinus floor and also displacement and destruction of the floor of the orbit and also causes the invasion and perforation of the cortical bone and metastasizes locally to the surrounding soft tissues. Radiographically, our case presented as cortical plate destruction with the invasion into the parapharyngeal space and also involving left masseter muscle simulating a benign neoplastic variant of the odontogenic tumor.

\subsection{Radiographic Features:}

It presents as small ovoid or round radiolucent areas on conventional plain radiographs. Unilocular radiolucency with smooth well defined margins, bilocular or multilocular with scalloped margins sometimes with ill-defined periphery surrounded by thick sclerotic margin. Minimal medullary expansion in the mesiodistal aspect cannot be seen in normal radiographs but perforation can be seen as focal discontinuity of the cortical border with the absence of sclerosis at the peripheral margin. The desquamated keratin contents may appear as cloudy or milky-way lumen[10]. The OKCs seen bilaterally or in both the jaws in patients younger than 10 years of age should give the suspicion of Gorlin-Goltz syndrome. The cortex of the lesions usually appears scalloped, and the borders are sclerotic. These lesions expand at the expense of the medullary space. Expansion of the cortices is more often seen in maxillary lesions, in which perforation of the floor of the maxillary sinus and the buccal cortex and nasal fossa may occur.

On MRI, OKCs typically have a low to intermediate signal intensity on T1-weighted images, and they have mixed hyperintensity and isointensity to hypointensity on T2-weighted images and do not enhance after Gd-DTPA administration. The cyst contents appear on T2-weighted images as hyperintense with areas of inhomogeneity or "signal drop out" that corresponds to the keratinaceous debris within the lumen[11,12].

\subsection{Histological Features:}

There are certain characteristic histopathological features that are gold standard guiding the pathologist and infact clinician to diagnose it as KOT according to Pindborg and Hanse ${ }^{[12]}$ which are as follows:

i. The epithelial lining is very thin and uniform in thickness, friable and often difficult to procure at the time of biopsy with little evidence of rete ridges.

ii. The basal squamous epithelial layer is prominent and palisaded with the thin spinous layer and intracellular edema.

iii. Keratinization is predominantly parakeratotic with nuclei but may be orthokeratotic layer and is corrugated.

iv. The fibrous connective tissue wall is generally thin and inflammation is absent.

v. There is a tendency of the epithelium to separate from the connective tissue lining and sometimes "abtrofung" or dropping down phenomenon of epithelial elements in the connective tissue wall is also seen[10]. 
The above features may closely resembles low grade squamous cell carcinoma which sometimes can be justified by the review of few incidence studies showing the transformation of KOT into intraosseous sqaumous cell carcinoma with a range of $0.1 \%-1.8 \%$ with a mean age of 60.2 years and a male:female ratio of 2.2:1[13]. The only criterion that proves that a squamous cell carcinoma has arisen from a KCOT is the demonstration of direct transition from the normal epithelial lining to invasive carcinoma. OKCs recur more frequently than any other jaw cysts at a rate of approximately 5\%. However there are wide variations in the recurrence ranges as per the literature[14]. The underlying mechanism involved in its recurrence are many;

i. Multiplicity of the lesion and the presence of satellite cysts causing difficulty in the surgical excision.

ii. Incomplete enucleation owing to the thin fragile epithelial lining with inherent intrinsic growth potential, increased mitotic activity which shows them as benign neoplasms.

iii. Scalloped margins of the lesion and multilocularity with the evidence of perforation and internal spiculation of the bony wall. A relationship between perforation of the lingual plate of the mandible and recurrence after treatment also has been observed[10].

iv. Cystic lesions arising from the basal cells of the oral mucosa also called as basal cell hamartias, particularly in the third molar region and ascending ramus of the mandible. They are the source of perforation of the overlying bone and firm adhesion of the cysts to the overlying mucosa and it is advisable that when the cysts were surgically removed, the overlying mucosa should be excised with them in an attempt to prevent possible recurrence, or the formation of new cysts, from residual basal cell proliferations.

Ironically, the enigmatic presentation of this tumor enabled many researchers in exploring many new strategies in diagnosis and treatment aspects of it macroscopically and also at the molecular level. Immunohistochemical studies shows positivity with PCNA, Ki67, p53 consistent with the higher level cell proliferating activity in the lining epithelial cells thus supporting the neoplastic nature in contrast to other keratinizing and non-keratinizing odontogenic cysts. An epithelial-specific cell surface glycoprotein, gp38 and carcinoembryogenic antigen (CEA) expression in the basal and suprabasal epithelial cells of the odontogenic keratocyst indicates altered gene expression suggestive of malignant potential.

\subsection{Treatment:}

The treatment strategies vary with the clinical presentation of the lesion, biologic nature and recurrent potential. The conventional options include:

i. enucleation and curettage,

ii. enucleation and peripheral ostectomy

iii. osseous resection without (rim ostectomy/ marginal resection) or with (segmental resection) continuity defect.

iv. Aggressive treatment modality like enucleation with application of carnoy's solution.

PTCH1 is an important molecule in the Hedgehog $(\mathrm{Hh})$ signaling pathway. This pathway is a key regulator of embryonic development controlling cell proliferation and cell fate. PTCH1 inhibits the signaling pathway by repressing the activity of Smoothened (SMO), So the loss of PTCH1 function by inactivating PTCH1 mutations as well as aberrant activation of SMO by activating SMO mutations could cause constitutive, ligand-independent signal transduction that may lead to neoplastic growth. Recent studies related to the PTCH1 pathway have provided some insights into the development of molecular therapeutic approaches for Gorlin syndrome and its related sporadic tumors, including OKCs. The Hh pathway can be blocked at different levels, and Hh inhibitors could serve as attractive anti-tumor agents. The intracystic injection of a SMO proteinantagonist may be the most promising treatment option[15].

\section{Conclusion:}

Keratocystic odontogenic tumor due to its indifferent clinicopathologic profile ensures thorough diagnosis and thereby dictates an ideal treatment option to prevent its recurrence which is the characteristic of it in view of a successful outcome. Although diagnosis can be confirmed by routine histopathology and radiography, it is worthwhile switching over to advanced molecular studies for more accurate surgical options especially with syndrome associated and recurrent lesions in the present clinical scenario. CT and MRI gives excellent details about surgical margins of the lesion including perforation with invasion to surrounding soft tissue structures. It is fortunate that genetic studies targeting the underlying molecular pathways may be valuable in the near future. 


\section{Figures:}

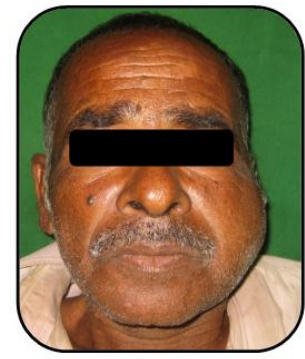

Figure1 Preoperative extraoral photograph

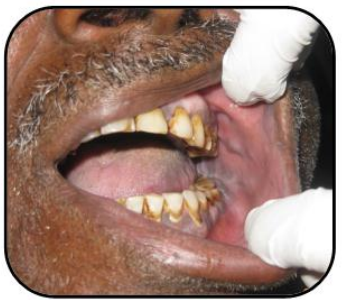

Figure 3 Preoperative intraoral photograph

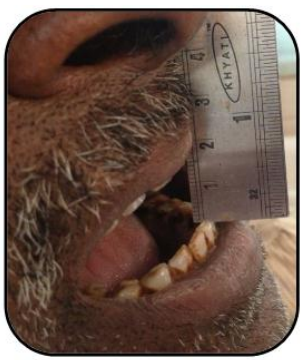

Figure 2 Limited mouth opening

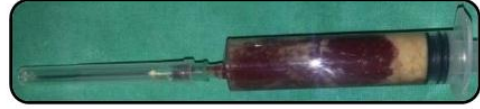

Figure 4 Aspiration

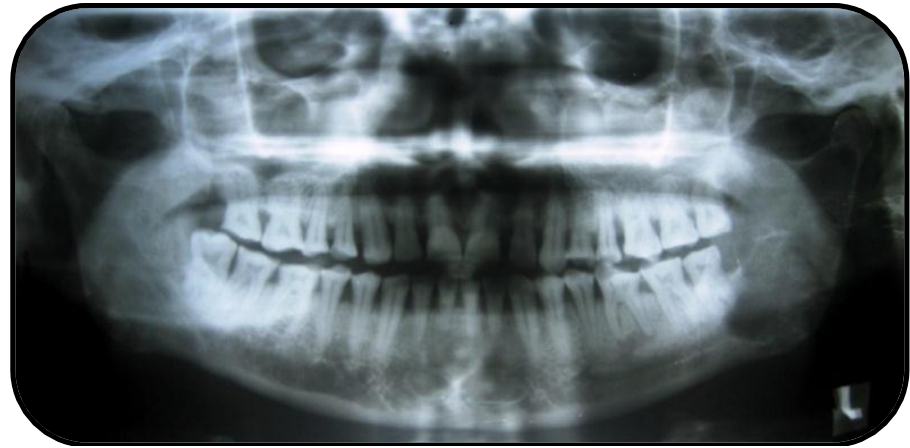

figure 5 orthopantomogram
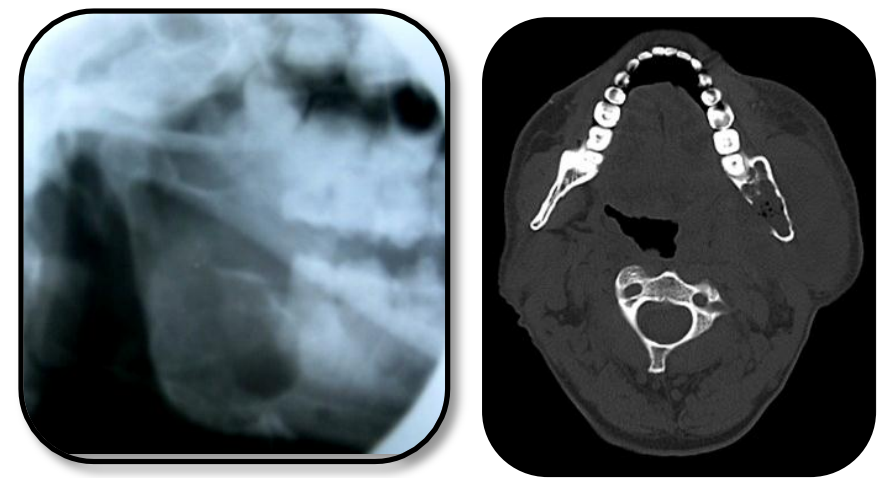

figure 6 lateral oblique view

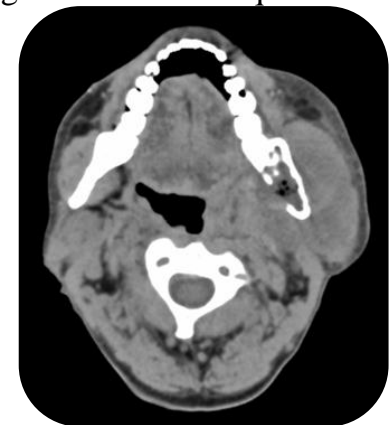

figure 8 contrast ct (axial view)

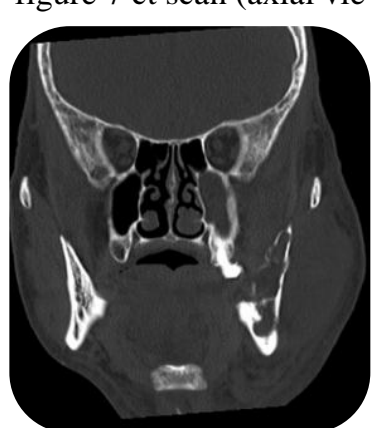

figure 9 ct scan (coronal view) 


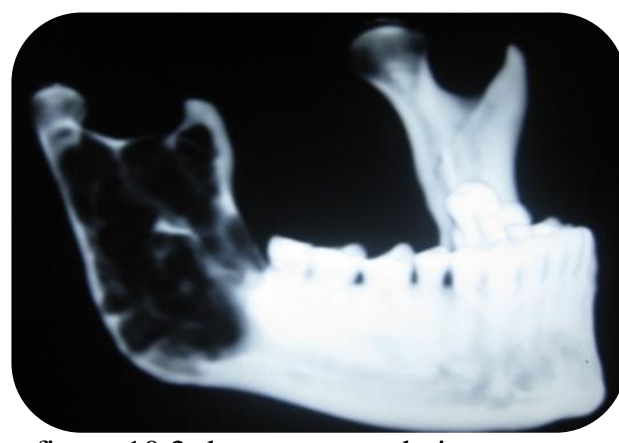

figure 103 -d reconstructed view

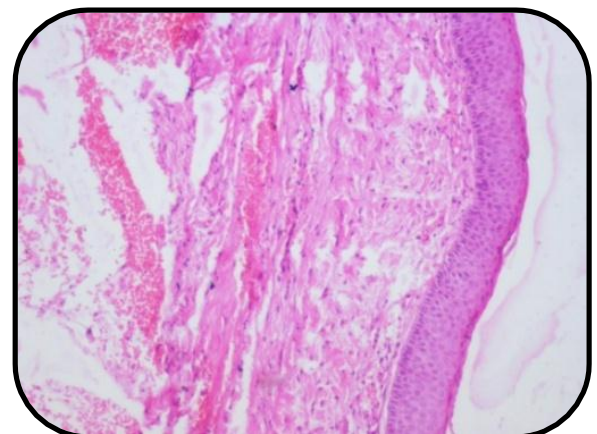

figure 11 histopathology

\section{References:}

[1] HE Veenstra-Knol, JH Scheewe, GJ van der Vlist et al., Early recognition of basal cell naevus syndrome, Eur J Pediatr, 164, 2005, 126-130.

[2] M Manfredi, P Vescovi, M Bonanini, S Porter, Nevoid basal cell carcinoma syndrome: a review of the literature, Int J Oral Maxillofac Surg,(33) 2004, 117-124.

[3] JV Soames, JC Southam, Oral pathology, 4th ed. Oxford: (Oxford University Press; 2005).

[4] L Barnes, JW Eveson, P Reichart, D Sidransky, Pathology and genetics of head and neck tumours. WHO classification of tumors series, Lyon: (IARC Press; 2005).

[5] BW Neville, DD Damm, Allen CM and Bouquot JE. Odontogenic cysts and tumors in Oral and Maxillofacial Pathology., $3^{\text {rd }}$ edition, 2009, pp. 683-691.

[6] G Ochsenius, A Ortega, L Godoy, C Penafiel, E Escobar, Odontogenic tumors in Chile: a study of 362 cases, J Oral Pathol Med, 31, 2002, 415-420.

[7] REL Gehani, M Orafi, M Elarbi, K Subhashraj, Bening tumours of orofacial region at Benghazi, Lybia: a study of 405 cases, J Cranio-Maxillofacial Surg, 37, 2009, 370-375.

[8] T Eryilmaz, S Ozmen, K Findikcioglu, S Kandal, M Aral, Odontogenic keratocyst: an unusual location and review of the literature, Ann Plast Surg, 62, 2009, 210-212.

[9] RI MacLeod, JV Soames, Squamous cell carcinoma arising in an odontogenic keratocys, Br J Oral Maxillofac Surg, 26(1), 1988, 52-57.

[10] JJ Pindborg, J Hansen, Studies on odontogenic cyst epithelium, 2: clinical and roentgenological aspects of odontogenic keratocysts, Acta Pathologica Microbiologica Scandinavica, 58, 1963, 283-294.

[11] RP Langlais, OE Langland and CJ Nortje, Multilocular Radiolucencies, (Williams \& Wilkins, 1995), Chapter-13, Diagnostic Imaging of Jaws, pp. 327-335

[12] B Tan, TS Yan, L Shermin, KC Teck, PC Yoke, C Goh, A Balakrishnan, Malignant transformation of keratocystic odontogenic tumor: Two case report, American journal of otolaryngology-head and neck medicine and surgery, 34, $2013,357-361$.

[13] G Borg, G Persson, H Thilander, A study of odontogenic cysts with special reference to comparisons between keratinizing and nonkeratinizing cysts, Swed Dent J, 67, 1974, 311-325.

[14] LJ van Rensburg, CJ Nortje, I Thompson, Correlating imaging and histopathology of an odontogenic keratocyst in the nevoid basal cell carcinoma syndrome, Dentomaxillofac Radiol, 26(3), 1997, 195-199.

[15] L Zhang, ZJ Sun, YF Zhao, Z Bian et al., Inhibition of SHH signaling pathway: molecular treatment strategy of odontogenic keratocyst, Med Hypotheses, 67, 2006, 1242 - 1244. 